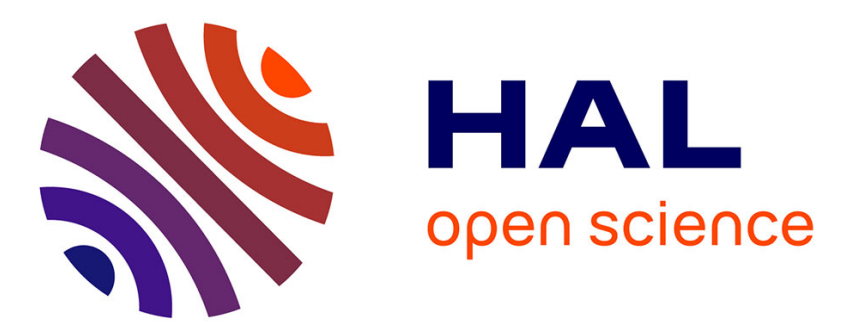

\title{
Quelles mathématiques faut-il apprendre pour se préparer à répondre aux questions des élèves et à traiter leurs erreurs? Le cas des commencements de l'algèbre
} Alain Jacques Mercier

\section{- To cite this version:}

Alain Jacques Mercier. Quelles mathématiques faut-il apprendre pour se préparer à répondre aux questions des élèves et à traiter leurs erreurs? Le cas des commencements de l'algèbre. Journées mathématiques de l'Institut Français de l'Education, IFE ENS-Lyon, Jun 2011, Lyon, France. hal01997155

\section{HAL Id: hal-01997155 \\ https://hal.science/hal-01997155}

Submitted on 28 Jan 2019

HAL is a multi-disciplinary open access archive for the deposit and dissemination of scientific research documents, whether they are published or not. The documents may come from teaching and research institutions in France or abroad, or from public or private research centers.
L'archive ouverte pluridisciplinaire HAL, est destinée au dépôt et à la diffusion de documents scientifiques de niveau recherche, publiés ou non, émanant des établissements d'enseignement et de recherche français ou étrangers, des laboratoires publics ou privés. 


\title{
Quelles mathématiques faut-il apprendre pour se préparer à répondre aux questions des élèves et à traiter leurs erreurs?
}

\section{Le cas des commencements de l'algèbre}

\author{
Alain Mercier* \\ ENS-Lyon IFE \\ 16 rue PASCAL \\ 13007 MARSEILLE \\ Adresse mail : alain.mercier@ens-lyon.fr
}

\begin{abstract}
RÉSUMÉ. Cet exposé repose sur le travail collectif des enseignants chercheurs (Yves Matheron, responsable du projet, Alain Mercier, Nadia Douek et Robert Noirfalise) et des 80 professeurs des équipes du projet AMPERES conduit par la Commission Inter-IREM " Didactique " et l'INRP. Il présente des résultats partiels mais cherche à rendre compte de ce que nous avons appris et démontré dans le cadre de la direction de plusieurs travaux doctoraux, qui ont donné lieu à publication: un enseignement non formel du travail algébrique est possible, mais cela suppose la constitution d'une culture collective des professeurs de mathématiques qui n'est pas formée dans le cadre de leurs études, quel que soit leur niveau de formation. En effet, cette culture ne relève pas des pratiques universitaires des mathématiques.
\end{abstract}

ABSTRACT.

MOTS-CLÉS : commencements de l'algèbre, situations didactiques, outils symboliques, modélisation algébrique, systèmes d'équations, savoir professionnel.

KEYWORDS :

Titre de la manifestation - Journées mathématiques IFÉ-ENS de Lyon 2011

Luc Trouche, Hamid Chaachoua, Magali Hersant, Yves Matheron et Giorgos Psycharis (dir.) (2012). Faire ensemble des mathématiques : une approche dynamique de la qualité des ressources pour l'enseignement, Actes des journées mathématiques de l'IFÉ, juin 2011, ENS de Lyon.

http://ife.ens-lyon.fr/editions/editions-electroniques/actes-des-journeesmathematiques-de-life

Un lien vers l'enregistrement de la conférence donnée est disponible sur ce site 


\section{Introduction}

Nous appellerons « commencements de l'algèbre » ce qui, dans les programmes français, appartient au calcul littéral et dans le socle commun porte sur les expressions du premier degré ou sur des programmes de calcul. C'est l'initiation à ce que nous appelons le travail algébrique sur des formes qui sont supposées dénoter des nombres.

Chacun de ces termes, qu'il soit venu des textes officiels ou du travail épistémologique, prendra chair au fur et à mesure de l'exposé. Mais déjà, on peut en imaginer le sens en pensant à la géométrie euclidienne comme au travail géométrique sur des figures qui elles aussi sont supposées dénoter des grandeurs ou des nombres, et au lien entre les deux que faisait Descartes, appelant géométrie l'ouvrage où il expose les notations (devenues définitives ou presque) du travail algébrique.

\section{ELEMENTS D'UN ETAT DES LIEUX}

Voici ce que l'on trouve dans les programmes de Quatrième, classe traditionnelle de l'entrée dans un travail algébrique explicite. On y lit par exemple : « Le calcul littéral qui a fait l'objet d'une première approche en classe de cinquième, par le biais de la transformation d'écritures (nous soulignons), se développe [...] en veillant à ce que les élèves donnent du sens aux activités entreprises dans ce cadre, en particulier par l'utilisation de formules issues des sciences et de la technologie (id). " Cependant, le texte définissant les attendus du « socle commun » se démarque : « dans le domaine du calcul littéral, les exigences du socle ne portent que sur les expressions du premier degré à une lettre et ne comportent pas les techniques de résolution algébrique ou graphique de l'équation du premier degré à une inconnue. »

L'ensemble des textes est composé en Times romain ou Times New Roman, corps 10, minuscule, interligné 12 points exactement, sans césure dans le texte, avec un alinéa de première ligne de $5 \mathrm{~mm}$. Un espace de 6 pts est défini après chaque paragraphe (format>paragraphe>espace après : $6 \mathrm{pts}$ ). Veiller aux lignes seules en bas et haut de page (format>paragraphe $>$ enchaînement $>$ éviter veuves, orphelines). Le style à utiliser est nommé « Corps de texte » dans ce document.

Les titres et sous-titres font l'objet d'un traitement particulier, expliqué ci-après. Par ailleurs, les auteurs qui désirent mettre en valeur un terme peuvent le faire en utilisant l'italique, mais jamais le soulignement ni le gras. Éviter au maximum d'utiliser les lettres majuscules dans les titres et dans le texte.

\subsection{Les textes institutionnels pourraient ouvrir des possibles}

En principe, un programme ouvre les possibles mais les professeurs (et souvent les corps d'inspection) les referment en enseignant seulement les exigences du socle 
(ce qui souvent élimine la plupart des objets de l'écosystème des exigibles et qui est une des causes d'échec repérée par les recherches sur ces questions. Les lettres figurant dans les formules et que l'on peut traiter comme des paramètres en font l'intérêt : que signifierait $\mathrm{v}=\mathrm{d} / \mathrm{t}$ (la vitesse est égale au rapport de la distance au temps), $\mathrm{C}=\mathrm{pQ}$ (le coût est égal au produit du prix unitaire par la quantité), $\mathrm{U}=\mathrm{RI}$ (la tension est égale au produit de la résistance et de l'intensité) ou $\mathrm{S}=\mathrm{eN}$ (la surface occupée par la foule est égale au produit de l'espace occupé par chacun par le nombre des personnes rassemblées), si on restreint ces «formules issues des sciences » à des « expressions à une seule lettre » et qu'on ne peut les considérer comme des modèles dont on peut tirer la résolution algébrique ou graphique de l'équation qui rend compte de la question « quelle est la distance parcourue en trois heures ? ", respectivement « le coût de douze tables de classe, la résistance connaissant l'intensité mesurée, ou le nombre de participants à un concert en plein air qui s'est tenu sur un terrain de trois hectares est-il plutôt de 20000 ou de 5000 ? (PISA 2000) » Les élèves ont le droit d'avoir été confrontés à de telles questions, qui selon certaines interprétations mobilisent des compétences n'appartenant pas aux exigibles du socle. Leurs parents et leurs professeurs devraient l'exiger.

Le résultat d'une interprétation réductrice des attendus du programme d'études, c'est que l'approche proposée aux élèves est finalement celle d'un système formel et c'est seulement a posteriori qu'un sens peut être cherché, pour des manipulations apprises formellement: ce que les attendus cités ici montrent déjà en nommant «calcul littéral» le domaine de pratiques auquel il s'agit d'initier les élèves, « lettres» les paramètres, variables, inconnues désignant des grandeurs, et «transformation d'écritures» le calcul permettant d'extraire une inconnue, d'exprimer une grandeur comme fonction des autres, de démontrer que deux formules sont équivalentes.

C'est pourquoi nos observations vont montrer que les programmes du Collège se paient de mots : « À travers la résolution de problèmes, la modélisation de quelques situations et l'apprentissage progressif de la démonstration, les élèves prennent conscience petit à petit de ce qu'est une véritable activité mathématique : identifier et formuler un problème, conjecturer un résultat en expérimentant sur des exemples, bâtir une argumentation, contrôler les résultats obtenus en évaluant leur pertinence en fonction du problème étudié, communiquer une recherche, mettre en forme une solution.» ou encore «.../... Au collège, est visée la maîtrise de techniques mathématiques élémentaires de traitement (organisation de données, représentations, mises en équation) et de résolution (calculs et équations bien sûr, mais aussi constructions). Leur emploi dans la prévision et l'aide à la décision est précieux dans de multiples circonstances, de la gestion familiale à l'activité scientifique ou professionnelle. » De fait la description précise du travail à faire contredit ces déclarations d'intentions : «1.2. Expressions littérales [Thèmes de convergence] : Utiliser une expression littérale. Produire une expression littérale. » l'idée des formules modélisant une classe de problèmes s'est perdue au profit d'une forme abstraite dont on pourrait avoir l'usage : «De nombreux thèmes du programme, notamment dans le domaine grandeurs et mesures, conduisent à utiliser des expressions littérales (formules) [...] apprendre à choisir et interpréter l'écriture 
appropriée d'un nombre ou d'une expression littérale suivant la situation » et voici que les expressions littérales se trouvent opposées aux nombres, ayant perdu leur force dénotative. La suite le confirme : « - apprendre à effectuer des transformations simples d'écriture ; - initier à la notion d'équation. » ce qui signifie : «-*Tester si une égalité comportant un ou deux nombres indéterminés est vraie lorsqu'on leur attribue des valeurs numériques. *La classe de cinquième correspond à une étape importante avec le travail sur des égalités vues comme des assertions dont la vérité est à examiner. La notion d'équation ne fait pas partie du socle commun. » Plus la censure est forte plus la description du travail devient pédante puisque voici que « tester si une égalité comprenant des nombres indéterminés est vraie » qui est la tâche des élèves revient selon le commentaire à « travailler sur des assertions dont la vérité est à examiner ». Pour sûr, personne ne doute que ce soit bien plus accessible que de découvrir que « si vingt mille personnes se tenant debout occupent 3 hectares alors chacune occupe $\mathrm{e}=\mathrm{S} / \mathrm{N}=3 \times 100 \mathrm{~m} \times 100 \mathrm{~m} / \mathrm{N}=30000 \mathrm{~m}^{2} / 20000=3 \mathrm{~m}^{2} / 2$ $=1,5 \mathrm{~m}^{2}$ soit (en moyenne) un rectangle de $1,5 \mathrm{~m} \times 1 \mathrm{~m}$, ce qui rend l'assertion crédible $»$.

Et en Quatrième le développement suit la même logique et on peut lire : « Le travail proposé s'articule autour de trois axes :

- utilisation d'expressions littérales donnant lieu à des calculs numériques ;

- utilisation du calcul littéral pour la mise en équation et la résolution de problèmes divers ;

- utilisation du calcul littéral pour prouver un résultat général (en particulier en arithmétique).

... À la suite du travail entrepris en classe de cinquième, les élèves sont familiarisés avec l'usage des priorités et avec la gestion d'un programme de calcul utilisant des parenthèses. En particulier, la suppression des parenthèses dans une somme algébrique est étudiée. L'objectif reste de développer pas à pas puis de réduire l'expression obtenue. Les identités remarquables ne sont pas au programme. Les activités de factorisation se limitent aux cas où le facteur commun est du type a, ax ou x2. " Cela interdit de fait les formules dont l'intérêt est d'être paramétrées et donc, de donner des modèles généraux pour de vastes catégories de problèmes, méritant ainsi d'être étudiées.

\subsection{Dans les manuels puis les classes, Factoriser est défini formellement}

"C'est transformer une expression, de la forme d'une somme à celle d'un produit »; "C 'est l'opération inverse du développement »; "C'est écrire une expression sous la forme d'un produit de facteurs » écrivent les manuels(Abou \& Mercier, s. d.). C'est donc une opération sur une forme, dont la présentation sur des exemples est faite par le professeur qui ainsi « la démontre ». Cette démonstration d'une manière conventionnelle de faire peut être répétée indéfiniment, et on observe qu'elle s'accompagne d'une sorte de commentaire qui n'arrive ni à justifier les manipulations réalisées ni à en donner le contrôle. En effet, la question de ce que les 
expressions expriment (ou plutôt, dénotent) n'est pas posée et ne pourrait l'être dans les termes qui sont proposés. On ne dit même pas qu'on transforme une somme (de produits) en un produit (de sommes) et que ce n'est pas toujours possible ! Aussi le professeur, dans la classe, ne trouve pas de manière convenable pour dire ce qu'il y a à faire car l'idée d'une technologie ou d'une théorie justifiant les techniques qu'il démontre lui est devenue étrangère. Ainsi, Abou Raad et Mercier observent qu'ils disent : " entre les deux on a un moins, avant et après on a un carré, on est donc arrivé à la troisième égalité remarquable » ou « Il n'y a pas de double produit, alors c'est a deux moins b deux » et encore «Il y a trois termes, c'est une des deux premières identités, j'écris une parenthèse et un carré à l'extérieur et pour le signe, je regarde le terme du milieu. » Ce genre de discours centré sur le bon comportement est observé systématiquement dans les classes des collèges, en France comme au Liban.

On peut dire que les professeurs inventent des discours non mathématiques pour nommer des pratiques supposées mathématiques. Or leurs manières de dire devraient faire fonction de théorie pour les formes que les élèves apprennent à manipuler, d'autant plus qu'on voit là tout ce que sera, pour la majorité de la population, l'expérience du travail algébrique. Or ces discours ad hoc ne sont pas contrôlés sur leur consistance et leur origine nous est inconnue. Les professeurs ont donc, pour l'enseignement du calcul des expressions algébriques, un problème symétrique de celui des élèves ; le problème des professeurs produit le problème des élèves.

Nous reconnaissons donc ici les caractères de la transmission par l'usage des manières de faire : " y'a rien à savoir! » vous dit-on, puisque « il n'y a qu'à faire ! » Depuis toujours, celui qui sait « démontre » la bonne combinatoire des assemblages en faisant le travail : il démontre le travail (ici, algébrique) comme un ensemble de manipulations formelles qu'il peut faire ; il montre qu'il anticipe les effets de son action (ici, la forme des écritures, en traçant par avance les parenthèses et les signes opératoires). Dans l'enseignement mathématiques, la plupart des notions (et des manières de les mobiliser dans des formes langagières) utiles au travail des notations (et des manières de représenter des objets et des relations pour en faire les objets d'un calcul) sont donc aujourd'hui des objets présentés par leur usage. Nous explorons ce phénomène qui pèse sur l'enseignement tout autant que naguère l'appel systématique au travail axiomatique de la réforme de 1970. Il est mondial.

Mais poursuivons l'exploration rapide engagée et son analyse. Les éléments d'un discours non mathématique accompagnent et portent l'action. Comme personne ne peut agir de manière efficace sans avoir les moyens de parler de ce qu'il fait, ce discours fonde les premières tentatives d'action des élèves. Professeurs et élèves nomment les objets sur lesquels porte l'action (il y a trois termes, j'aurai un carré avec un signe), ainsi que les relations qui sont expérimentées dans cette action (c'est la première identité). Mais si professeurs et élèves ne disposent jamais des termes mathématiques pour penser ce qu'ils font, ils inventent des termes et des descriptions ad hoc (pour le signe je regarde le terme du milieu) qui peuvent augmenter les difficultés : ainsi, cette bonne gestion des signes et des termes renforce les comportements de « conservation de l'information ostensive » dont on a 
montré (Tonnelle, 1979) qu'ils fondent les erreurs de manipulation algébrique qui perdurent des années durant. De plus, si ces manières ne sont pas consistantes, on voit les professeurs pris, de temps à autre, d'une " bouffée de rigueur " inefficace car 1) elle est trop ponctuelle et 2) elle les conduit vers encore plus de formalisme maladroit 3) ils apparaissent alors aux yeux des élèves comme des professeurs psychorigides, rien de plus (Rouy, 2007). Si on jouait vraiment le jeu d'un enseignement formaliste, alors le savoir visé serait par exemple celui qu'a formé Serfati(Serfati, s. d.), et qui est exposé ici : les assemblages formels doivent être bien formés. Si c'est le cas ils peuvent dénoter un nombre (ou tout autre un objet relevant d'un calcul) ou bien être utilisés pour démontrer une propriété générique. L'auteur décrit ainsi les pratiques relatives aux assemblages de symboles algébriques : «ce que nous appelons écriture ou expression est un assemblage symbolique ; l'assemblage bien formé de deux signes chiffrés comme 14+23 dénote un nombre, dont le signe chiffré est 37. » Et plus loin « $(2 p-1)+(2 p+1)$ ne dénote aucun nombre mais peut être réduit à $4 \mathrm{p}$. Cette réduction démontre que la somme de deux impairs consécutifs (...) est multiple de 4. » Si l'action est absolument formelle alors le langage pertinent doit être contrôlé sur sa consistance, puisqu'il fait fonction de théorie. Et Serfati montre comment Leibnitz procède à partir des possibilités qu'offrent les notations cartésiennes. Un enseignement formel assumé porterait sur les assemblages bien formés et leurs usages. Pour autant, qu'on nous comprenne bien, nous ne pensons pas que la solution des difficultés des élèves et de leurs professeurs soit dans l'introduction de la description produite par Serfati et nous cherchons donc une alternative. Le fait que le problème des élèves ait été identifié dans le monde entier, par la répétition des mêmes erreurs, et que le problème d'enseignement demeure, montre que la solution n'est pas évidente. Mais nous avons appris a minima qu'il faut interpréter un assemblage, pour dire ce qu'il dénote, ou pour comprendre ce qu'il démontre.

Afin de comprendre que les assemblages peuvent dénoter tout objet calculable et non pas seulement des nombres, nous allons examiner un cas simple et connu. Lorsque $\mathrm{a}$ et $\mathrm{b}$ désignent le déplacement positif d'une case selon une ligne ou une colonne, dans un quadrillage orienté, et n le nombre de déplacements, la « formule du binôme $»$ rend compte des divers déplacements de $n$ cases : $(\mathrm{a}+\mathrm{b}) \mathrm{n}=\mathrm{an}+\mathrm{nan}-1 \mathrm{~b}$ $+\ldots+$ Cni an-ibi $+\ldots+$ bn. L'assemblage symbolique dénote donc les déplacements positifs de $n$ cases selon $n$-i lignes et i colonnes, pour toutes les valeurs de $\mathrm{i}$, de 1 à $\mathrm{n}$, et il permet de les compter. Ainsi, la formule est un outil combinatoire bien plus générique que nous l'aurions pensé en la considérant comme « développement de la puissance nième d'une somme de nombres ». De même, a et b peuvent dénoter les tirages pile et face du jeu et cela donne une fonction caractéristique de la distribution de probabilité. Étudier ce qu'un même assemblage peut dénoter, et quels sont les systèmes de règles de manipulation que permettent les notations algébriques (qu'on pourrait comparer par exemple aux propriétés des notations géométriques), ne peut être un enjeu que dans un travail universitaire, mais ce serait la question posée dans un enseignement de combinatoire qui suivrait les chemins que nous allons proposer plus loin sur le travail algébrique : les possibles que permet de penser le travail de modélisation.

Titre de la manifestation - INRP 2011 
Mais le Collège n'est pas le seul lieu d'un discours réduit à accompagner le faire. Pour diriger et penser la calcul de la différence «75-28», en deuxième année de l'enseignement primaire, les professeurs disent « 8 moins 5 est impossible, on emprunte donc 1 au 7 de gauche et on peut alors faire « 8 moins 15, 7 » que l'on écrit dans les unités; il reste alors à faire la retenue. Les élèves écrivent :

$$
\begin{array}{cccc}
75 \text { qui devient } & 7613 \text { et enfin } & 7 & 13 \\
-28 & \frac{-2}{4} & 8 & \frac{-2+1}{4} \\
& & 4 & 8
\end{array}
$$

Nous rejoignons ici les observations sur le calcul numérique élémentaire, où se produit le même phénomène que pour le calcul algébrique (Ma, s. d.). C'est l'ensemble de l'enseignement des mathématiques qui a perdu ses énoncés technologiques et théoriques et qui est réduit à ce que Chevallard appelle «une praxis sans logos $»$ : une pratique muette. Le phénomène est mondial ou presque et Ma montre par exemple que pour enseigner comment faire la soustraction 49-17, on soustrait parallèlement dizaines et unités et on dit (aux USA comme en France) « 9 moins 7, 2 » que l'on écrit comme unités, et « 1 moins 4, 3 » que l'on écrit comme dizaines. Jusque là rien à dire sinon que, pour 73-25, l'usage du même schème conduit les professeurs à dire « 3 moins 5 est impossible, on emprunte donc un aux 7 de gauche et on peut alors faire et dire « 13 moins 5,8 » que l'on écrit comme unités. Mais il faut se rappeler que l'on a "emprunté 1 à 7 » et donc " faire une retenue ». Deux manières sont alors proposées : soit, « de 7 reste 6 » et on continue alors « 6 moins 2,4 » pour les dizaines, soit, " on retient ce 1 qu'on ajoute en bas », pour dire « 2 et un de retenue, 3 moins 7, 4 ». Plusieurs questions sont ainsi sans réponse : 1) Quand et comment rend-t-on l'emprunt ? 2) Pourquoi retient-on le $1 \mathrm{de}$ 15 qu'on a emprunté à 6, en l'ajoutant en bas? 3) Pourquoi les deux manières sontelles équivalentes? C'est pourquoi la technique la moins incompréhensible va l'emporter, mais c'est aussi la manière de faire impraticable : tentez donc une division en écrivant des soustractions faites ainsi, vous devrez poser les soustractions et bientôt vous n'aurez plus la place pour écrire les retenues.

La pratique « de retenue » ne se comprend que si l'on peut penser que l'on a décomposé 75 et non pas que l'on a emprunté à 7 . De ce fait on ne pense pas 75 comme dénotant « 7 dizaines et 5 unités » mais « 6 dizaines et 15 unités », ce qui ne change pas la différence avec 28 mais permet de la calculer. C'est ainsi que procèdent les professeurs, en Chine. On peut alors penser que l'on transforme également les deux termes de la soustraction ce qui ne change pas le résultat, tandis que le discours sur l'emprunt décrit juste une manipulation formelle.

\subsection{Ce type d'enseignement par dressage produit des idées erronées et des apprentissages lents}

Dans ces conditions, les élèves s'essaient donc à faire sans savoir, ils tâtonnent et généralisent sans contrôle. Les erreurs que l'on observe élèves sont les mêmes en tous pays au début du travail algébrique, comme nous l'avons noté plus haut et par exemple ils proposent avec insistance la transformation connue : $a 2-b 2=(a-b) 2$ 
qui conserve toute l'information que porte l'écriture puisque l'on retrouve les lettres a et $b$, le signe -, l'exposant 2. La mise en commun de 2 entre a et b revient donc aux étourdis, comme un mauvais pli. Et pire, les élèves peuvent bien en être persuadés, puisqu'ils y voient la transformation introduisant les parenthèses rondes, connue d'eux sous le nom de "distributivité ». Tonnelle a appelé le principe qui rend compte de cette manière " la conservation de l'information ostensive ». Ce même principe fonde aussi les explorations initiales de l'écriture par les enfants, qui la comprennent d'abord comme représentation des choses (ainsi, le mot pour puce doit être plus petit que le mot pour rat, ou encore, le ma de maman n'a rien à voir avec celui de malotru)...

Ce type d'enseignement conduit à des apprentissages si lents qu'il est fort peu efficace : la plupart des gens s'y refusent et oublient rapidement tout cela, les autres apprennent une mécanique formelle qu'ils ne peuvent que difficilement retravailler pour en faire évoluer le sens ou les manières, puisqu'ils n'en disposent pas comme d'un savoir (Mercier, 1992). Ce type d'enseignement relève d'une manière didactique antique, proche de l'apprentissage par cœur: la restitution correcte ne garantit pas l'appropriation d'un savoir utilisable, le pouvoir de faire

\section{PEUT-ON IMAGINER UNE AUTRE VOIE, NON FORMELLE, ET INTERVENIR ?}

Nous développons des travaux dans le même esprit sur tous les niveaux, de la maternelle à la Troisième. Nous avons travaillé longtemps dans notre séminaire d'équipe (de 2003 à 2006) sur ce que nous avons appelé « les représentations 》 au sens large (Goody, 1997), avec l'idée que les œuvres culturelles qui nécessitent des écoles sont des représentations (et les techniques de production de représentations) et les théories (qui permettent de penser ce que sont les représentations).

Ces représentations (en mathématiques, ce sont des systèmes de notations) sont des ajouts au monde, des créations humaines sur lesquelles la pensée s'appuie. C'est ce qu'on appelle en mathématiques le calcul dans les modèles. Ainsi la pensée n'est plus en rapport immédiat au monde, et au delà de la langue, dont l'expérience montre les insuffisances, la pensée s'outille d'artefacts, les représentations organisées en modèles d'un domaine de réalité. Ces représentations sont souvent objets de méfiance parce que les modèles ne rendent pas compte de tout. Comme artefacts simplificateurs, ils fausseraient le rapport à la complexité des choses. On interprète ainsi une différence entre le rapport culturel à l'algèbre et à la géométrie. Ce qui empêche d'ailleurs certains de penser que la géométrie propose elle aussi un travail dans un modèle de l'espace et des nombres, sur une feuille de papier, et non pas un travail dans l'espace même, comme certains l'affirment. Afin de sortir du dilemme, nous affirmons que les représentations représentent non pas le monde, mais les idées que notre action dans le monde nous conduit à former sur le monde, idées qui fondent ainsi nos stratégies d'action. J'y reviendrai, car cela explique qu'on ne puisse pas enseigner directement la manipulation formelle des représentations. 
Le problème, c'est que la tendance spontanée de l'enseignement est de « naturaliser » les représentations pour surmonter l'obstacle, tandis qu'il faudrait rendre les représentations à leur vérité d'outils culturels de la pensée, inventions humaines au même titre que le tournevis, la perceuse, la machine à laver le linge, le téléphone portable, tous objets que l'on croit pouvoir utiliser sans y penser ou presque tandis qu'il faut une école pour acquérir une compréhension même sommaire de leurs principes et être capable d'en comprendre le bon usage ou de suivre le travail de leur dépannage. Nous allons donc imaginer la genèse artificielle des connaissances algébriques scolaires sur le modèle générique d'un type d'objets qui a fait irruption dans la culture commune avec les usages de l'informatique, les jeux de console. Ceux-ci répondent en effet à certaines des conditions données par les théorisations de l'action en situation didactique, et ils fournissent donc une métaphore efficace pour les élèves comme pour les professeurs :

- le joueur s'y oppose à un milieu dénué d'intentions mais organisé, dont il apprend les propriétés pour y agir ;

- l'organisation des tableaux construit une progression en mettant en jeu la survie du joueur, qui en cas d'échec peut cependant rejouer du début.

De ce fait, l'idée permet de réinterpréter la théorie des situations (Brousseau, Balacheff, Cooper, Sutherland, \& Warfield, 1997) en repensant les propriétés d'une « situation d'action » comme celles d'un « jeu vidéo par tableaux ». Tous deux définissent un milieu où agir qui permet par ses rétroactions de juger de la réussite de l'action, et qui réserve ainsi la possibilité d'une stratégie exploratoire « de tâtonnement ». La différence tient bien sûr dans l'enjeu, qui est dans un cas la seule réussite de l'action et dans l'autre l'apprentissage d'un savoir. C'est pourquoi les situations didactiques ne sont pas seulement des jeux dans un milieu mais des jeux sous contrat didactique. Cela demande l'intervention d'un professeur, dont l'action a pour objet la désignation de l'enjeu didactique de la situation d'action, ce qu'il fait à la fois en jouant sur les variables de cette situation et en désignant explicitement cet enjeu comme savoir à apprendre.

\subsection{La banque de problèmes permet de désigner aux élèves un type de tâche à étudier}

L'organisation des problèmes en « tableaux » permet de donner aux élèves la responsabilité de la résolution d'un type de problèmes (Chevallard, 2007), dont on organise l'exploration en tableaux de difficulté croissante. Pour notre premier exemple (banque de problèmes modélisables par un système de deux équations à deux inconnues) en 3ème, au début de l'année, les élèves ne disposent que connaissances arithmétiques et de quelques techniques relatives aux équations du 1er degré à une inconnue. Nous devons imaginer que peut-être ils ne disposent d'aucune expérience de formules de calcul autre que celle des calculs d'aire $(a=1 / 2$ bh), de volume $(\mathrm{v}=4 / 3 \pi \mathrm{r} 3)$, de vitesse $(\mathrm{v}=\mathrm{d} / \mathrm{t})$, formules qu'ils n'ont manipulées qu'en « remplaçant les lettres par des valeurs » comme les instructions officielles le demandent. 
Quels sont donc les principes d'usage d'une banque de problèmes à visée didactique ? La donnée simultanée de plusieurs problèmes permet au dispositif d'engager les élèves vers l'épreuve de leur stratégie de première invention et donc, de ne pas les reprendre sur celle-ci. C'est sans doute une difficulté pour les professeurs, qui n'ont pas pour habitude de laisser des élèves s'embarquer dans des modèles qu'ils jugent aberrants en attendant que la suite des évènements détrompe les élèves. C'est pourtant ce qu'il va se produire. En revanche, le débat entre élèves est proposé à tout moment, entre élèves travaillant sur les mêmes problèmes, et c'est la comparaison de l'efficacité des modèles proposés qui fera la sélection des modèles pertinents et des stratégies efficaces. Le professeur peut cependant intervenir sur le travail d'un élève, chaque fois qu'il le juge utile, mais surtout le professeur organise la rencontre des élèves avec les situations que les problèmes de la Banque évoquent, en proposant différents moments de l'étude.

On peut parler en ces termes du processus qu'il conduit : a) Pour chacun des niveaux de l'étude, le professeur organise, dans la classe, les conditions d'un débat qui permettra à chaque élève de passer progressivement de l'idée qu'il aura eue et de l'action qu'il aura mise en œuvre lors d'une phase de recherche personnelle à une manière de faire plus assurée.

b) Chaque élève aura éprouvé l'efficacité de sa manière, parce qu'il l'aura confrontée et, peut-être, partagée avec les autres élèves d'un groupe de travail. Il considérera ainsi la manière de faire qu'il se propose comme une stratégie d'action.

c) Le groupe des élèves accédera ainsi à une technique - une manière efficace qui sera devenue traditionnelle et qui aura été validée lors d'une présentation à toute la classe et d'un débat entre les groupes.

d) Chaque élève pourra alors éprouver personnellement la pertinence de cette technique, dans le traitement des problèmes sur lesquels lui et son groupe auront échoué dans un premier temps, juger de l'efficacité que procure son usage, poser les problèmes que pose sa mise en œuvre dans des situations nouvelles.

La banque de problèmes est présentée au professeur en ces termes :

Chacun des problèmes peut être résolu par un élève qui ne disposerait d'aucune technique, parce qu'il est possible d'en chercher une solution par tâtonnements. Les élèves sont en état de débattre de la validité des stratégies qu'ils proposent, parce qu'ils savent valider leurs réponses à partir de la connaissance qu'ils ont acquise par tâtonnements. La classe de problèmes définit un domaine de pratiques capable de fonder le savoir technique que ces procédés portent. Il est toujours possible d'en chercher une solution par tâtonnements exploratoires. Le tâtonnement est en effet une stratégie d'exploration, la plus efficace en l'absence d'informations sur le domaine exploré. La donnée de plusieurs problèmes de difficulté graduée permet rapidement aux élèves de produire des stratégies de plus haute technicité : nous rendrons compte plus loin de cette propriété. Pour que les élèves apprennent des mathématiques, le travail du professeur est de faire évoluer le rapport des élèves aux problèmes. Le premier moment conduit les élèves à proposer des formes langagières qui orientent leur action, et à mobiliser des outils 
symboliques qui permettent le calcul. Le professeur y veille. Dans un deuxième moment qui vient rapidement, le professeur enseigne en interprétant ces productions et en orientant les élèves vers le traitement formel du problème. A cet effet il leur donne des éléments théoriques qui leur permettent d'interpréter leurs trouvailles efficaces comme des productions mathématiques pertinentes.

Le fait que les problèmes étudiés soient linéaires garantit que le tâtonnement produira une première connaissance du mode de vie du problème. C'est ainsi que les techniques arithmétiques de (double) fausse position sont pour cette classe de problèmes des stratégies optimales de tâtonnement, culturellement identifiées et mises en forme. Cependant la culture algébrique (minimale, mais existante) des élèves et l'intervention du professeur, qui oriente les élèves vers l'étude des compte rendus écrits de leurs stratégies en les faisant d'abord communiquer par groupes puis, en leur proposant la rédaction d'un transparent pour exposer à la classe, évitent cette impasse qu'est l'arithmétique : le raisonnement ne doit pas risquer de remplacer la modélisation algébrique. Nous savons que celle-ci est rendue possible parce que nous disposons d'une théorie de la formation des systèmes symboliques mathématiques qui nous donne les étapes du mouvement : des formules numériques de la forme $« 2$ lits $\times 5$ chambres +8 chambres $=18$ personnes logées $»$ apparaissent d'abord comme moyen de vérifier qu'une première idée selon laquelle 5 chambres à deux lits et huit chambres à un lit permettent de loger 20 personnes est plausible mais fausse, et l'usage systématique de ces écritures montre un format répété de questionnement qui peut s'écrire $2 \mathrm{x}+\mathrm{y}=20$ et être l'objet de tentatives de traitement algébrique pour des élèves qui ne sont pas ignorants du travail des équations et disposent donc de quelques éléments pratiques de substitution ou de transposition. Enfin, grâce à l'organisation explicite de la Banque en tableaux, le professeur peut à tout moment relancer l'intérêt des élèves pour l'étude des techniques de résolution dont ils disposent. Il peut en effet proposer des problèmes semblables qui posent des difficultés nouvelles, organiser l'étude des diverses stratégies et des discours justificatifs qui leur sont associés, dans les divers temps de débat. La banque garantit en effet que les élèves sont en état de débattre de la validité des stratégies qu'ils proposent, parce qu'ils savent valider leurs réponses à partir de la connaissance qu'ils ont acquise par tâtonnements : ils n'ont donc pas à attendre l'évaluation du professeur et peuvent progresser par eux-mêmes des couples de valeurs solutions. Ainsi, l'accès à la banque peut être libre, et ne pas être limité au temps de classe. (Elle pourrait tout aussi bien être mise en ligne que, comme dans notre observation, donnée en version papier). L'organisation didactique de cet accès libre est un acte d'enseignement et à ce titre, il engage la responsabilité du professeur qui indique aux élèves la matière de l'étude à conduire après le travail en classe. Voici donc le texte initial distribué aux élèves et lu collectivement :

Avertissement Les problèmes proposés ici relèvent tous d'une même classe, ce qui signifie qu'il existe une méthode générale de résolution de tous ces problèmes. Ils sont proposés à votre étude afin que, en cherchant une méthode pour en résoudre un, vous vous fassiez une idée des mathématiques qui sont au programme puis, en cherchant à les résoudre tous avec l'aide de vos camarades et sous la direction de votre professeur, vous puissiez découvrir par vous même une

Titre de la manifestation - INRP 2011 
partie des mathématiques au programme de votre classe.

Ces problèmes sont choisis de telle manière que vous puissiez résoudre certains d'entre eux avant même de connaître une méthode mathématique valable pour tous : pour chacun d'eux, vous pouvez vérifier par vous même si votre réponse est juste. Cependant, leur difficulté graduée augmente de tableau en tableau avec votre expérience et vos connaissances : vous pouvez donc tenter de les résoudre tous d'emblée ou, au contraire, vous pouvez attendre que le travail collectiffait en classe vous ait donné accès à des méthodes éprouvées et mathématiquement reconnues. Vous emporterez chez vous ce cahier, pour chercher une solution aux problèmes que vous n'auriez pas résolu en classe ou pour étudier à loisir comment utiliser la méthode inventée par un autre élève ou par un autre groupe : vous pourrez ainsi vérifier si une méthode est valable pour tous les problèmes ou si elle échoue dans certains cas.

L'annonce explicite de l'enjeu du travail proposé définit donc une situation, pour les élèves comme pour le professeur. L'idée que les mathématiques sont une invention permettant de résoudre des types de problèmes et non pas des problèmes isolés, aléatoirement rencontrés, est ici essentielle. Ces classes de problèmes relèvent de grandes questions, qu'en général on n'identifie pas. Le professeur doit le savoir, pour " garder le cap » et ne pas prendre de décisions contraires au mouvement engagé. La résolution des systèmes d'équations fait partie des grandes questions qui sont l'horizon de l'étude et on déclare ici que les mathématiques du programme permettent de traiter de cette question vaste, et effectivement : 1) On s'engage dans un domaine des mathématiques dont l'étude durera plusieurs années, avec l'algèbre linéaire et les questions géométriques qui sont ainsi modélisées. 2) On développe des techniques d'attaque qui ont un grand avenir en calcul, la formalisation et la généralisation du tâtonnement linéaire fondant la plupart des techniques du calcul numérique.

Le commentaire indique que toutes les idées sont bonnes à suivre. Nous verrons l'effet de cette déclaration avec le travail du premier élève que nous présenterons. La question de la vérification du résultat d'une stratégie personnelle est une des conditions de son existence. L'annonce de leur graduation permet enfin à un élève de s'arrêter en chemin et d'attendre, pour bénéficier des avancées des autres. Cela garantit le progrès collectif (on remarquera que c'est une stratégie didactique absolument opposée à l'enseignement individualisé : c'est que " savoir » est la propriété d'un collectif).

Les quatre problèmes du premier tableau sont les suivants :

Premier problème

A la clinique " la Sauvegarde ", il n'y a que des chambres à un lit et des chambres à deux lits. Aujourd'hui la clinique est complète : vingt malades occupent tous les lits des 13 chambres. Combien de chambres à un lit et de chambres à deux lits y a-t-il à la Sauvegarde ?

Deuxième problème Un grand hôtel dispose de 50 chambres et peut recevoir 83 personnes. Il y a des chambres pour une personne et des chambres pour deux

Titre de la manifestation - INRP 2011 
personnes. De combien de chambres pour une personne et de combien de chambres pour deux personnes dispose cet hôtel ? Troisième problème Dans un refuge de montagne, il n'y a que des chambres à deux lits et des chambres à 4 lits. Aujourd'hui elle affiche complet, 30 randonneurs occupant tous les lits des 12 chambres du refuge...

Quatrième problème Dans une colonie de vacances il y a des dortoirs de 5 lits et des dortoirs de 7 lits. Il y a 79 dortoirs et 469 enfants dans la colonie, où il n'y a plus un lit libre.

Dans cette colonie de vacances, combien y a-t-il de dortoirs à 5 lits et de dortoirs à 7 lits ?

On observe que les élèves commencent à chercher en utilisant diverses techniques personnelles, en recourant à des dispositifs ostensifs très variés. Nous trouvons un éventail étendu de ces objets graphiques : dessin de chambres et lits, écritures en toutes lettres, flèches, traits, symboles mathématiques, etc. Certains de ces ostensifs (BOSCH \& CHEVALLARD, 1999) sont des symboles appartenant au monde du travail algébrique et ont été précédemment introduits en classe comme les symboles d'opérations : croix + trait - étoile $\times$ deux points : mais aussi comme le symbole d'égalité : deux traits $=$ et les symboles d'agrégation : parenthèse ouvrante (, parenthèse fermante), ou le trait de fraction et même, l'accolade \}. Les productions des élèves sont donc variées et le professeur peut suivre leur évolution. Nous présentons ci-dessous deux exemples de ce que l'on rencontre.

\subsection{Premières productions : Albert}

Pour Albert, au delà du tâtonnement, une première stratégie est portée par cette représentation, qui est iconique mais permet l'expérimentation : situer les chambres par le signe « ch » et placer sous chacune des traits représentant les lits des malades.

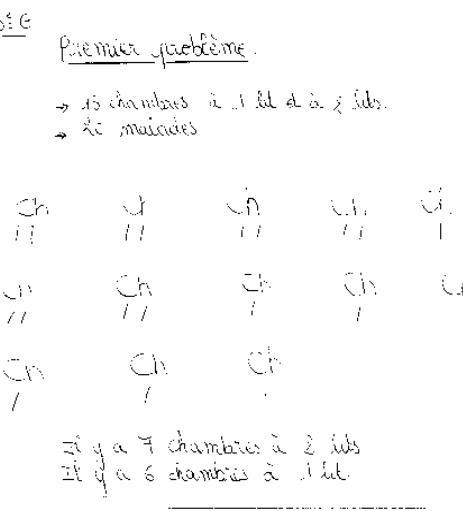

Nous avons vérifié que de très jeunes élèves réussissent à résoudre le problème par cette méthode, et notre commentaire initial a encouragé les élèves de $3 \mathrm{e}$ qui n'ont pas imaginé mieux à procéder ainsi. On remarque que cette représentation décrit « la distribution de 20 malades dans 13 chambres ", et donne une formule de vérification que nous qualifierons d'autant plus aisément de « rhétorique » qu'elle ne mobilise aucun symbole algébrique mais seulement les nombres en symboles chiffrés. La technique est efficace sur les deux premiers problèmes de ce premier tableau. Elle conduit Albert à développer une stratégie consistant à attribuer deux lits à chacune des chambres, avant de créer des chambres de quatre lits jusqu'à obtenir le nombre total de lits de l'énoncé.

Titre de la manifestation - INRP 2011 
C'est une stratégie qui n'est déjà plus un tâtonnement bien qu'elle ne puisse être qualifiée de fausse position. Elle permet en effet un calcul direct, que nous observerons pour le problème suivant : 12 chambres à deux lits par chambre, cela fait 24 lits; le nombre de lits restant est $30-24=6$, il reste donc à fabriquer 3 chambres à 4 lits. S'il fallait qualifier cette technique nous dirions qu'elle appartient donc plutôt au

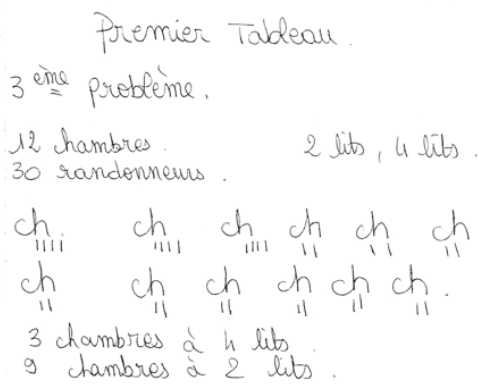
monde du raisonnement combinatoire.

\subsection{Premières productions : Bernadette}

Pour Bernadette, le tâtonnement est organisé par sa présentation sur la feuille, et

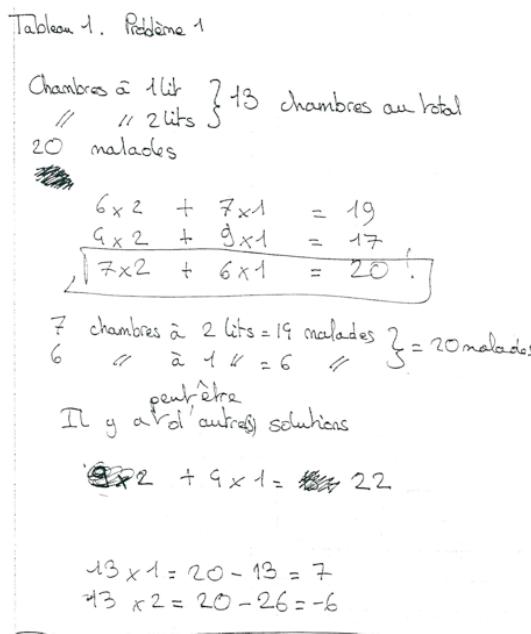
l'usage immédiat de la formule numérique mobilisant les symboles $\times,+,=$. Les meilleurs élèves commencent ainsi. On remarque aussi une disposition des écritures tentant de rendre compte des deux contraintes de l'énoncé en utilisant les deux directions du plan, ce qui sera atteint au deuxième essai. L'accolade sépare alors les raisonnements sur les chambres et sur les malades, et en montre les liens entre les deux énoncés qui déterminent les contraintes du problème.

L'élève conduit l'exploration à partir de cette représentation,. La formule en ligne est donc un acquis central, que le professeur aura à faire diffuser en organisant rapidement un exposé des diverses manières de traiter le problème que porteront les divers groupes d'élèves. La forme rhétorique qui est utilisée pour énoncer une première réponse est donc liée à Tableau 1 . Prodiene 2

donc une forme de type algébrique, calculable, permettant l'exploration : un modèle du problème. Cependant, la présentation $\mathrm{du}$ résultat montre l'usage rhétorique du signe $=$, pris comme verbe d'un énoncé.

Le tâtonnement de Bernadette n'est pas organisé d'emblée par la linéarité de la relation, mais par la deuxième contrainte à respecter et qui est notée au dessus des

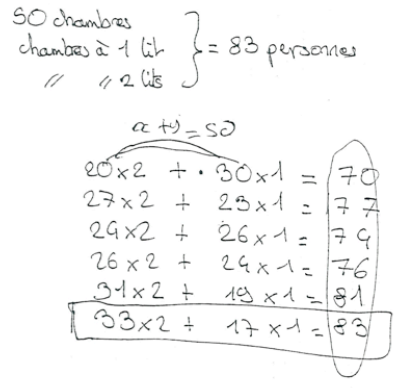

Titre de la manifestation - INRP 2011 
écritures exploratoires.

La découverte de la régularité des variations sera, pour Bernadette, une conséquence non immédiate de son exploration systématique. Bientôt, elle aura intégré que la fonction est croissante avec le nombre de chambres de chaque sorte.

Peut-être cette connaissance sera-t-elle encore locale : elle s'énoncerait alors « le résultat est 50 de plus que le nombre de chambres à 2 lits ", et cela engagera vers une compréhension plus profonde de la situation, quand ce sera confronté à la stratégie d'Albert.

Le professeur qui cherche à accompagner les élèves au plus près doit garder en mémoire les diverses connaissances ainsi construites, et les élèves qui en sont porteurs, dans l'exploration de la situation.

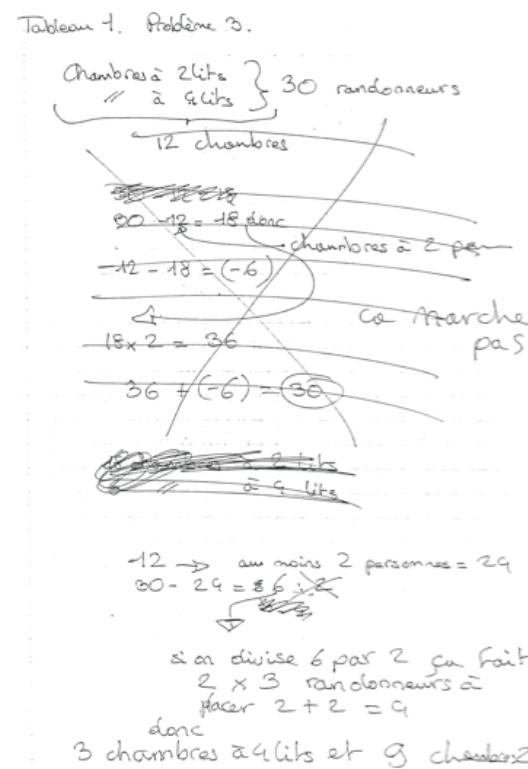

$\mathrm{Au}$ troisième problème, Bernadette pense les deux équations avec deux accolades horizontale et verticale, mais cherche à sortir de la stratégie de tâtonnement. Elle imagine donc une suite d'opérations conduisant directement à un résultat : un algorithme. Mais si l'idée qu'elle met en œuvre (et qui est présente chez Albert) fonctionnait immédiatement dans le cas précédent (distribuer les randonneurs soit, remplir des chambres en y casant un randonneur ce qui permet de caser 12 randonneurs, et justifie une soustraction) elle doit être ici adaptée car on doit remplir des chambres à 2 lits et donc attribuer 2 randonneurs à chaque chambre (24 randonneurs sont ainsi placés) afin de bénéficier de l'information « il reste 6 randonneurs à placer, ils finiront de remplir 3 chambres à 4 lits ». On le comprend, le travail est toujours un raisonnement, les écritures algébriques ne sont pas manipulées formellement mais sous le contrôle de leur interprétation rhétorique. Nous verrons bientôt l'importance de ce raisonnement, qui apparaît chez des élèves fort différents.

\subsection{Le travail d'enseignement : le jeu sur les variables de la situation}

Après la mise en commun des trouvailles de chacun dans des groupes dont l'enjeu était la production d'un transparent et la présentation à la classe du travail des 6 groupes de 4 élèves, lecture est donnée d'un « commentaire » qui ensemble fait la théorie de l'activité conduite et propose un premier prolongement à l'activité.

Commentaire 1, lu après le premier tableau

Titre de la manifestation - INRP 2011 
a) Pour vérifier vos réponses, vous avez progressivement appris à écrire des formules qui notent, à partir des calculs correspondant au problème, l'égalité qu'il faut obtenir. Ces formules expriment les relations entre les grandeurs dont parle l'énoncé du problème, que l'on appelle des variables. L'ensemble des égalités qui doivent être vérifiées est un modèle de la situation que l'énoncé expose. Vous pouvez écrire un modèle de chacun des quatre problèmes du premier tableau : pour cela vous utilisez chaque fois deux variables, et vous pouvez voir un phénomène nouveau pour vous : la solution d'un de ces problèmes vérifie deux formules à la fois.

b) Pour chercher les valeurs des variables qui vérifient ces formules, on peut les considérer comme des équations : pour disposer d'une technique algébrique générale de résolution de ces problèmes, vous devez donc apprendre à résoudre ensemble deux équations qui comprennent chacune deux inconnues. Or, vous savez transformer une formule à l'aide des règles du calcul algébrique, il s'agit donc maintenant d'apprendre à faire systématiquement les transformations qui aboutissent à la réponse.

c) Vous pouvez reprendre chacun des problèmes du premier tableau : vous avez inventé des manières de les chercher qui peuvent vous aider à imaginer des calculs algébriques intéressants et vous pouvez vérifier vos calculs puisque vous connaissez les valeurs des variables qui sont les solutions du problème. Mais vous pouvez aussi tenter de résoudre les problèmes du deuxième tableau par des calculs et des raisonnements algébriques, puis, vérifier vos calculs après coup, en testant les réponses auxquelles vous aboutissez.

La première partie du commentaire pose le problème nouveau de la classe : utiliser un modèle de la situation pour produire la réponse en cherchant la solution. Elle engage d'abord les élèves à reprendre leur travail sur les 4 problèmes de ce premier tableau, pour tester les nouvelles connaissances qu'ils peuvent mobiliser maintenant, ce qui permet à tous de rattraper le mouvement. Cela suppose que le professeur et les élèves reconnaissent comme formule des écritures du type de celles que l'on peut observer : aussi bien « $33 \times 2+17 \times 1=83$ et $33+17=50$ » que « 33 chambres à 2 lits et 17 chambres à 1 lit cela fait 83 lits pour 50 chambres » ou que «

$$
33 \times 2 \text { lits }=66 \text { lits }
$$

$17 \times 1$ lit $\quad=17$ lits

50 chambres, 83 lits »

L'accord des élèves est aisé parce qu'ils ont effectivement utilisé de telles formes comme des formats, soit pour guider le tâtonnement soit pour énoncer la réponse. Mais le texte donné par le professeur va plus loin en nommant variables les grandeurs du modèle, qui peuvent varier pour donner selon le cas la formule numérique répondant aux contraintes (comme pour les exemples ci-dessus) ou une formule qui n'y répond pas (comme dans les tâtonnements des élèves). De ce fait, la notion de modèle peut prendre sens, à la fois comme forme générique des quatre problèmes proposés et comme forme spécifiée de chacun de ces quatre problèmes, permettant l'exploration formelle de l'espace des réponses possibles, exactes ou erronées. Le texte poursuit en identifiant deux variables pour deux formules, et en 
proposant de considérer cela comme système de deux équations à deux inconnues pour engager les élèves à écrire ces équations et à les manipuler par une extension praxémique (Matheron, s. d.) des techniques qu'ils connaissent pour les équations simples, à une seule inconnue. Le défi serait impossible à tenir s'il n'engageait les élèves à s'attaquer de nouveau aux quatre problèmes dont ils connaissent une solution, avant de vérifier que la nouvelle technique qu'ils auront inventée sera efficace sur les problèmes du deuxième tableau.

Le travail demandé est donc maintenant non plus la production de la réponse, mais la production de techniques de résolution des équations. L'enjeu n'est absolument plus le même et il n'est plus question, par exemple, de représenter les situations par un dessin : le travail est dorénavant dans le monde des représentations symboliques (Brousseau, 2005). Les élèves ont donc à réaliser une invention technique, qui s'appuiera sur le fait que dorénavant plus aucun élève ne s'imagine incapable de déterminer la solution, par un procédé certes primitif mais efficace : plus aucun élève ne peut donc affirmer : « je n'ai pas compris quel était le problème ». Le fait que le tâtonnement soit efficace n'est surtout pas mis en doute par le professeur (alors que l'on sait son inefficacité dès que les solutions sont non entières). En effet, la confiance des élèves en leur capacité à produire une solution est fondée sur une propriété essentielle des expressions algébriques : on peut vérifier une proposition de solution en cherchant le nombre que la formule dénote lorsque l'on attribue une valeur numérique aux signes, et cette action est consistante avec une compréhension rhétorique des assemblages symboliques. C'est donc le travail de la question posée maintenant qui conduira les élèves à une compréhension nouvelle, ce ne sont pas les explications du professeur.

Les instructions de la troisième partie du commentaire ont donc pour enjeu de montrer aux élèves que le travail algébrique qu'ils peuvent engager se fera sous le contrôle des résultats, qu'ils connaissent, et donc sous leur responsabilité. C'est pourquoi le commentaire est rédigé et doit être lu complètement, parce que les professeurs n'ont pas pour habitude de donner aux élèves la responsabilité de l'évaluation de leur travail, et qu'ils oublieraient trop souvent de décrire ainsi le contrat particulier de cet enseignement. Les élèves revisitent donc leur premier travail.

\subsection{Retour sur le premier tableau : Albert}

Après une mise en commun des trouvailles de chacun dans des groupes dont l'enjeu était la production d'un transparent et la présentation à la classe du travail des 6 groupes de 4 élèves, le commentaire a donc été lu.

On en voit les effets : Albert s'empare de la disposition de l'énoncé inventée par Bernadette et son groupe, puis il tente d'entrer dans un travail algébrique en nommant $\mathrm{x}$ et $\mathrm{y}$ les variables du problème, mais il n'arrive pas à une formule sur laquelle il puisse calculer. Il semble que l'accolade ne signifie pas pour lui l'exécution d'une somme et que l'écriture $1 \mathrm{x}+2 \mathrm{y}=83$ soit indispensable au passage à un calcul, en association avec $\mathrm{x}+\mathrm{y}=50$.

Titre de la manifestation - INRP 2011 

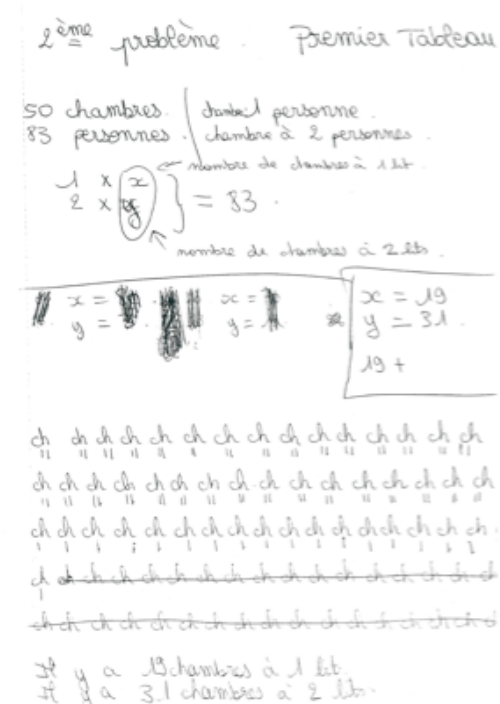

sans technique de vérification est de 50\%.

Albert s'attaque alors aux problèmes du deuxième tableau, pour voir, dirions nous en pensant toujours au mouvement d'exploration que le dispositif d'enseignement encourage. Et là, le dessin est rendu impraticable, non seulement parce que les nombres sont grands mais aussi parce que les « pièces de 5 » ne peuvent être représentées comme « contenant 5 pièces de 1 », tandis que les " chambres de 4» contenaient « 4 lits » (et donc, pas 4 chambres de 1 lit : la forme langagière différente interdit d'énoncer la question des pièces pour la représenter à l'identique de la question des chambres).

\section{Deuxième tableau}

Premier problème Dans mon porte-monnaie, il n'y a que des pièces à 1 euros et des pièces à 5 euros. Aujourd'hui j'ai 20 pièces et j'ai 56 euros. Combien ai-je de pièces de chaque sorte?

\section{Deuxième problème}

Dans mon porte-monnaie, il n'y a que des pièces à 2 euros et des pièces à 5 euros. Aujourd'hui j'ai 232 euros et j'ai compté 86 pièces. Combien ai-je de pièces de chaque sorte?

Les problèmes portent cette fois sur la valeur et le nombre de pièces de monnaie. Cela a pour but de disqualifier la stratégie de distribution des malades dans les

Titre de la manifestation - INRP 2011 
chambres, qui était efficace pour le premier tableau, et plus généralement toute stratégie de représentation iconique.

C'est de bonne guerre dans les jeux vidéo et cela ne devrait pas décourager Albert, qui va bientôt être éliminé du jeu et devoir reprendre son travail du début faute d'être entré dans l'apprentissage de la technique maintenant indispensable. Car effectivement, personne ne pense à « distribuer des euros à des pièces », pour savoir combien de pièces de 1 et 5 euros on doit avoir sachant qu'on a 56 euros avec 20 pièces.

Et en effet c'est à ce moment que Albert se décide à revenir au premier problème du premier tableau avec la technique des deux équations, qui a entre temps été présentée publiquement dans le commentaire et montrée au moins une fois à propos d'un des problèmes du premier tableau. Il écrira d'abord les formules de vérification avant de s'essayer au traitement des formules génériques tel que nous l'observons ici. Le dispositif fonctionne donc et bientôt, Albert écrit

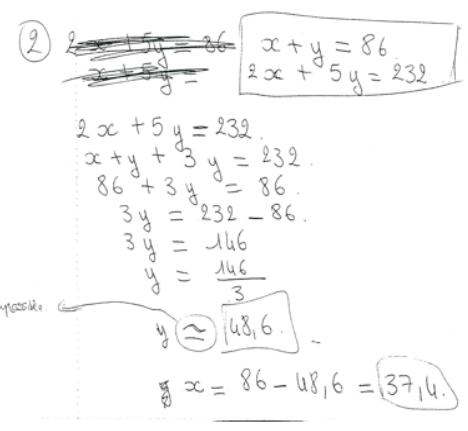
(difficilement) les deux formules qui modélisent la situation.

On le voit alors engager le travail sur ces formules et la stratégie est étonnante : il isole deux fois $\langle x+y$ » dans $2 x+5 y=232$ afin de le remplacer par 86 . Oublions son erreur (c'est $2(x+y)$ qu'il cherchait à isoler, et cela fait 172). Remarquons que sa technique produit en effet une transformation radicale du problème : il n'y a plus qu'une seule inconnue et Albert sait résoudre ce type d'équations. Même, il en déduira la valeur de l'autre inconnue.

On pourrait reconnaître là « le pivot de Gauss » mais il faut plutôt y reconnaître le raisonnement combinatoire de distribution dont nous parlions dans le cas de cet élève, à propos du deuxième problème (présenté plus haut). Nous pensons alors que ce calcul démontre que le travail algébrique de Albert est fondé sur la manière dont il a raisonné quand il a résolu ce type de problèmes sans outil algébrique.

Ce travail algébrique n'est pas, pour cet élève, un travail formel mais l'inscription d'un raisonnement : son écriture est sous le contrôle de ce raisonnement, qui est une théorie des manipulations possibles dans le monde dont les équations rendent compte. C'est en ce sens que nous pouvons donc affirmer que, pour Albert, la situation que nous lui avons proposée a fonctionné comme « une situation fondamentale pour le travail des systèmes d'équations ", au sens de Brousseau (1997). La situation peut être dite fondamentale, parce que les raisonnements que les élèves peuvent tenir dans le moment de l'action initiale peuvent fonder leur contrôle des représentations symboliques qu'ils écrivent et servent donc de théorie pour l'entrée dans ce travail si particulier des mathématiques que l'on appelle à tort « abstraction ».

Titre de la manifestation - INRP 2011 
Nous avons donc identifié l'ensemble des contraintes de l'enseignement des systèmes d'équations, en Troisième en France, et surtout, nous savons les conditions de réussite de cet enseignement. Cela suppose connues et maîtrisées les références et les travaux de didactique que nous avons cités, ils s'étalent sur trente ans et ont mobilisé au moins une centaine de chercheurs en épistémologie comme en didactique : ces savoirs ne sont pas toujours considérés comme appartenant de plein droit aux mathématiques, c'est pourtant tout cela qu'il faut savoir, pour enseigner de façon non formelle une question qui fait obstacle depuis que l'on cherche à enseigner ce type de travail c'est-à-dire depuis les " Lettres à une princesse d'Allemagne » de Monsieur Descartes. Nous allons maintenant montrer que nos affirmations sont fondées sur des observables.

\subsection{Du travail algébrique sur les formules à la résolution des équations}

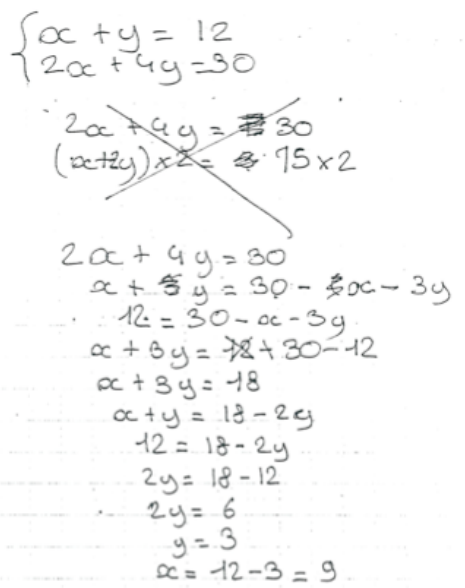

Le travail du professeur va maintenant consister à orienter les élèves vers la production de raisonnements algébriques capables d'accompagner leur travail sur les systèmes d'équations. On voit ici la reprise du deuxième problème du premier tableau, par Albert, dont nous suivons l'évolution.

L'élève tente une transformation formelle du système, écrit avec une accolade à la demande du professeur qui supportait mal le traitement séparé des deux équations. On remarque que la manière dont les accolades sont utilisées ne porte pas l'information ostensive que portait le modèle des élèves, tel qu'on peut l'observer ci-dessus chez Bernadette. C'est que le professeur et les traditions qu'il représente changent moins aisément que les élèves, qui apprennent tout ce qu'ils peuvent dans la situation qu'ils rencontrent. $2 x+4 y$ c'est bien $2(x+2 y)$ et $30,15 \times 2$. Albert aurait pu en conclure presque immédiatement que $x+2 y$ vaut 3 de plus que $x+y$ et donc, que $y=3$, mais c'eut été raisonner sur les équations et il cherche un traitement calculatoire qui lui interdit de penser ce raccourci.

Albert tente une autre transformation qui est elle aussi l'effet d'une comparaison entre les deux équations, mais qui se fonde sur l'interprétation de ces écritures comme des formules : il transforme la formule $2 x+4 y=30$ afin d'isoler $x+y$, ce qui lui permet de remplacer $\mathrm{x}+\mathrm{y}$ par sa valeur, 12, et comme cela n'élimine pas $\mathrm{x}$, il recommence ! Son calcul est exact. Que demander de mieux puisqu'il aboutit à la résolution de l'équation? 


\subsection{En conclusion}

On trouve ici enfin, sur la feuille de travail d'Albert, les deux formes du raisonnement qu'il a conduit, dans le cas $\mathrm{du} 4 \mathrm{e}$ problème du tableau 1 , qu'il reprend en fin de séquence. L'observation de cette page permet de confirmer nos interprétations : le raisonnement qui avait été rédigé maladroitement pour son quatrième emploi et formalisé comme « méthode plus simple » pour résoudre tous les problèmes de ce type, supporte le calcul conduit sur les deux formules qui modélisent le problème et qui met en évidence, justement, cette différence entre ce que Albert nommait A et a.

Ainsi, le travail algébrique a pu devenir, pour les élèves en difficulté

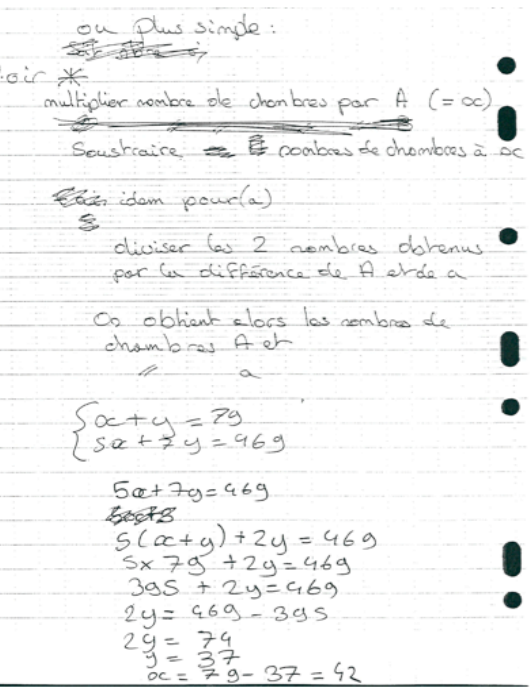
comme pour les meilleurs, un travail d'expression formelle de raisonnements.

Ce que nous observons n'est plus le travail de formes pures, vides de sens, que nous avons dénoncé en introduction. L'algèbre devient pour les élèves « La langue bien formée qui permet le calcul des raisonnements ", ce qu'elle était pour ses inventeurs.

Cela suppose seulement mais c'est beaucoup, car cet exposé ne montre qu'une toute petite partie des difficultés à surmonter, sur une question seulement et à un seul niveau d'enseignement au Collège, que les élèves peuvent constituer le raisonnement sur les catégories de problèmes qu'ils apprennent à résoudre avant d'engager le travail algébrique de production d'une technique.

C'est si loin de ce que l'on peut observer au quotidien, qu'il nous en faut une interprétation. Il n'y en a qu'une : les professeurs ne savent pas cela, parce que ce n'est pas ce qui leur a été enseigné et ce n'est pas ce qu'ils ont appris du travail algébrique. Ni au cours de leurs études secondaires, où ils ont pratiqué docilement un calcul automatisé, ni au cours de leurs études universitaires, où ils ont étudié l'algèbre, un domaine mathématique qui a peu à voir avec les problèmes que nous avons observé.

Les professeurs ne savent donc pas le chemin qui conduirait leurs élèves vers cette production et son identification complète par l'ensemble de la profession des mathématiciens de Collège qu'ils sont supposerait des études spécialisées bien plus développées que ce que l'année de formation professionnelle permet. Car le processus est loin d'être achevé : je n'en ai décrit que le commencement, l'enseignement auquel je réfère se développe sur trois tableaux encore, pour la classe de Troisième, et a des prolongements au delà. Et puis, il n'est pas entièrement satisfaisant: dès qu'ils calculent, les élèves n'écrivent plus aucun commentaire ni 
raisonnement... comment donc vont-ils maintenant décrire leurs calculs ? Comment le professeur pourra-t-il nommer les opérations qu'ils réalisent et qui manifestement ne peuvent être désignées par les deux propriétés axiomatiques qui parfois sont donnés (distributivité, conservation de l'égalité par soustraction d'un même terme aux deux membres). Par exemple, Albert isole l'expression $\mathrm{x}+\mathrm{y}$ dans $5 \mathrm{x}+7 \mathrm{y}$, substitue à $5(\mathrm{x}+\mathrm{y})$ sa valeur numérique $5 \times 79$ dans la formule, par ce procédé, il élimine la variable $x$ et produit une équation à une inconnue y qu'il résout avant de reporter la valeur de $y$ dans une des équations initiales afin de déterminer l'autre inconnue, x. Ces termes sont-ils disponibles, normalisés, permettent-ils de décrire les actions de calcul et pourraient-ils faire l'objet d'un enseignement visant à démontrer leur consistance?

Une des difficultés du mouvement didactique proposé, c'est que les questions des élèves vont très vite au fond des choses et que le professeur va prendre peur, ne sachant plus où les élèves vont l'emmener. Car ils vont en effet demander ce qu'il se passerait si on donnait trois équations, et il faudra alors leur montrer que trois équations à deux inconnues ne sont pas nécessairement compatibles, mais que la troisième équation qui permet de donner la réponse et que l'on s'est donnée donne une réponse stable tant qu'elle est combinaison d'équations compatibles aux deux premières. Quant on sait que : « [étudier] une technique systématique de résolution des équations du premier degré à une inconnue ne fait pas partie des compétences exigibles du socle commun » et que cette injonction n'est pas souvent interprétée par l'inspection à l'aune du dicton « qui peut le plus peut le moins », on comprend les professeurs qui renoncent à l'usage d'un outil d'enseignement efficace mais trop technique : les sociétés inventent et utilisent les moyens technologiques que leur culture moyenne leur permet.

\section{Discussion : ce que nous savons sur la question}

\subsection{Imaginer des situations pour faire émerger des représentations}

Nous appellerons notions des éléments de discours qui relèvent des expériences culturelles réalisées dans des domaines d'expérience. Les notations sont alors des objets substitutifs des objets du domaine d'expérience étudié comme domaine de réalité. Les notations rendent compte des pratiques dans ce domaine de réalité. Elles permettent des manipulations réglées (u calcul) sous le contrôle des notions et des discours associés, qui proviennent de l'expérience, et donnent un sens pratique au calcul. En ce sens, les notations représentent les propriétés des objets du domaine de réalité sur lesquels l'action se réaliserait.

En mathématiques, et surtout dans leur enseignement, les notations (des manières de représenter des objets et des relations pour en faire les objets d'un calcul) sont presque toujours données aux élèves comme des outils tout faits, qu'il suffirait d'apprendre à mettre en œuvre. Cela crée de nombreuses difficultés et des quiproquos dont l'exploration a été longue et difficile, je vous en ai rapidement présenté les résultats les plus significatifs.

Titre de la manifestation - INRP 2011 
Le problème est alors le suivant : de même que les outils mobilisés dans les pratiques quotidiennes ont une fonction de représentation de l'action, c'est leur sémioticité, ces outils désignent (à qui les connaît) l'activité qu'ils outillent ; ils désignent (à qui en connaît par expérience l'usage) la technique mobilisée pour résoudre le problème que l'activité rencontre, les notations montrent donc « ce que l'on pourrait faire ». Les notations, comme les outils, ne montrent cela qu'à qui connaît culturellement et personnellement leur usage. C'est un cercle vicieux comme on l'a vu pour le travail algébrique, mais il en va de même pour tous les apprentissages silencieux, par dressage sur le tas ou à l'école.

Une école devrait avoir d'autres ambitions que d'organiser l'apprentissage du travail algébrique par un dressage de plusieurs années. Mais il ne suffit pas de le dire et d'imaginer une voie de réponse, il faut aussi développer les infrastructures nécessaires à l'appropriation sociale de la technique qui résout la question : comme le transport aérien, qui suppose les aéroports, l'enseignement proposé ici suppose une culture universitaire productrice et porteuse de ces connaissances. La responsabilité de la situation n'appartient plus, aujourd'hui, aux IUFM et aux formateurs qui étaient sur le point de découvrir ces phénomènes en travaillant les questions didactiques qu'ils observaient. Elle appartient aux universitaires, dont on a supposé que leur manière de produire des mathématiques allait les conduire à savoir tout cela tout naturellement. Nous verrons bien, il suffit d'observer patiemment comme nous le faisons depuis quarante ans, à l'INRP et partout où des didacticiens travaillent, et de continuer à montrer que le chemin est connu, mais qu'il faut le viabiliser.

\subsection{Comprendre le travail d'enseignement}

Quelques résultats en didactique, dont nous avons montré l'usage :

1) Pour nous, il faut donc appuyer l'enseignement sur des expériences culturelles ou anthropologiques(Dewey, 1958) ou (Boero et al., 1995). Nous appelons situations les conditions de ces expériences et quand elles sont artificielles scolaires nous les appelons Situations Didactiques avec Brousseau.

Le travail du professeur est alors de définir des situations permettant l'émergence de représentations, puis de faire vivre ces situations, pour que les élèves identifient les représentations produites et en explorent les propriétés (Brousseau, 2005). C'est manifestement le travail de toute une profession et non pas celui de chaque professeur en particulier. Les didacticiens travaillent sur le fait que les élèves peuvent produire par eux-mêmes des représentations, qu'ils peuvent faire évoluer vers les notations attendues si on leur propose des situations adaptées. Les didacticiens ont vérifié que ce faisant, les élèves produisent les notions correspondantes à la condition que le professeur en observe les prémisses et les reçoive en les nommant.

2) Nous avons montré par ailleurs (depuis vingt ans que nous observons les élèves de tous âges et niveaux engagés dans un processus d'apprentissage), que des expériences culturelles ou anthropologiques non scolaires fournissent aux élèves les 
métaphores (les éléments discursifs) pour l'interprétation des situations didactiques qu'ils ont à affronter lorsqu'on les enseigne. Ces métaphores sont fondatrices de leurs connaissances: nous les qualifions pour cela de fondamentales. Le professeur doit savoir y faire appel en mobilisant les jeux de langage qui ont été formés à leur occasion.

Le travail du professeur est alors d'interpréter les pratiques de mathématisation initiales des élèves. Pour enseigner, le professeur qui a organisé une situation initiale accompagne les élèves en reconnaissant publiquement les pratiques mathématiques émergentes des élèves, afin qu'elles aient un avenir et que les élèves puissent développer collectivement une pensée outillée et validée.

3) Ce qu'il faut savoir pour accompagner les élèves dans les apprentissages que l'enseignement demande, c'est donc ce que sont ces expériences fondamentales et quelles sont leurs conditions d'évolution. Nous cherchons maintenant à montrer que dans des conditions bien définies (des situations que les professeurs doivent connaître), les élèves (qui mobilisent les métaphores fondamentales que la situation évoque pour eux et que le professeur connaît) peuvent produire d'eux-mêmes les systèmes de notations dont ils ont besoin : mieux, ces systèmes sont ceux que l'on cherche à leur enseigner.

Le professeur en organise l'exploration, il nomme les objets ainsi produits et engage les élèves à développer des jeux de langage stables à leur propos pour accompagner le processus dans lequel les élèves s'engagent. Pour cela, il doit être au fait des moments nécessaires de toute situation didactique. Ces moments sont au moins trois, comme nous avons pu le voir sur l'exemple étudié :

1) la formation d'une expérience du problème,

2) la formalisation d'une représentation du problème,

3) l'étude et l'épreuve des propriétés de cette représentation.

En mathématiques, nos observations et nos travaux vont de la maternelle à la Terminale en passant par tous les niveaux intermédiaires (projets École Kleber, École Saint Charles, AMPERES). Nous montrons ici deux des moments du travail algébrique, en Troisième autour des systèmes d'équations à deux inconnues, ces moments correspondant environ à trois heures de travail en classe. Nous avons bien d'autres exemples, par exemple au début du Collège autour des premières écritures algébriques (Krysinska, Schneider et Mercier 2009), ou en Cinquième avec les programmes de calcul comme appui à l'introduction des relatifs (Matheron, Schneider et Mercier 2013). Nos travaux d'ingénierie consistent donc à développer les suites de situations 1) qu'un professeur puisse définir pour les élèves, 2) où un professeur puisse les accompagner parce qu'il en connaîtra les moments clés, les points d'appui et les enjeux épistémologiques : trois dimensions à décrire pour rendre nos productions robustes et fiables.

Les connaissances qu'un professeur doit mobiliser pour faire son travail sont multiples, elles relèvent de ce qu'appellerai, pour dire en une seule formule ce que j'ai tenté de montrer sur un exemple, une analyse épistémique des domaines 
d'expérience culturelle et des situations didactiques que ces domaines fondent. Des travaux en ce sens se développent, dans le monde (Shulman, 1986). Les auteurs qui réfèrent à Shulman appellent " Pedagogical Content Knowledge » les savoirs pratiques du professeur qui résultent de ces analyses, et Shulman affirmait, lors d'une conférence devant l'assemblée des professeurs de mathématiques des USA, que contrairement au dicton selon lequel « ceux qui savent faire font, ceux qui ne savent pas faire enseignent », il fallait bien comprendre que " ceux qui enseignent sont ceux qui ont une compréhension profonde de ce qu'il y a à faire. " J'affirme pour ma part, en complément de ce que dit Shulman, que pas plus que dans le cas des pilotes d'avion que dans celui des ingénieurs des travaux publics, « la formation de cette compréhension nécessaire ne peut être laissée aux hasards du développement de l'expérience professionnelle des professeurs. » Il y faut le travail collectif d'étude de ce domaine de réalité qu'est ce que nous appelons le didactique.

\section{Bibliographie}

Abou, R. N., \& Mercier, A. (s. d.). Etude comparée de l'enseignement de la factorisation par un facteur commun binôme, en France et au Liban. Recherches en didactique des mathématiques. Vol. 29. Num. 2. p. 155-188.

Boero, P., Dapueto, C., Ferrari, P., Ferrero, E., Garuti, R., Lemut, E., Parenti, L., et al. (1995). Aspects of the mathematics-culture relationship in mathematics teaching-learning in compulsory school. PME conférence (Vol. 1, p. 1-151).

Bosch, M., \& Chevallard, Y. (1999). La sensibilité de l'activité mathématique aux ostensifs: objet d'étude et problématique. Recherches en didactique des mathématiques, 19(1), 77-123.

Brousseau, G., Balacheff, N., Cooper, M., Sutherland, R., \& Warfield, V. (1997). Theory of didactical situations in mathematics 1970-1990. Kluwer academic publishers.

Chevallard, Y. (2007). Readjusting didactics to a changing epistemology. European Educational Research Journal, 6(2), 131-134.

Dewey, J. (1958). Experience and nature (Vol. 1). Dover Pubns.

Goody, J. (1997). Representations and contradictions: ambivalence towards images, theatre, fiction, relics and sexuality. Wiley-Blackwell.

Ma, L. (s. d.). Knowing and teaching elementary mathematics: Teachers' understanding of fundamental mathematics in China and the United States (Vol. 46).

Matheron, Y. (s. d.). Mémoire et Etude des Mathématiques. Une approche didactique à caractère anthropologique.

Rouy, E. (2007). Formation initiale des professeurs du secondaire supérieur et changement de posture vis-à-vis de la rationalité mathématique.

Titre de la manifestation - INRP 2011 
Serfati, M. (s. d.). La révolution symbolique.

Shulman, L. S. (1986). Those who understand: Knowledge growth in teaching. Educational researcher, 15(2), 4-14.

Tonnelle, J. (1979). Le monde clos de la factorisation au premier cycle. DEA, Universités de Bordeaux I et d'Aix-Marseille II.

\subsubsection{Pour les ouvrages}

Le titre doit être en italique et le reste en romain.

Krainer, K. \& Wood, T. (Eds.). (2008). Participants in Mathematics Teachers Education: Individuals, Teams, Communities and Networks, Vol. 3. Rotterdam/Taipei: Sense Publishers.

\subsubsection{Pour les revues et les actes de conférences publiés}

Le titre de la revue ou de la conférence doit être en italique et le reste en romain.

Coquidé, M., Fortin, M., Rumelhard, G. (2009). L’investigation : fondements et démarches, intérêts et limite. Aster, 49, p. 51-78.

Sanchez, E. (2009) Innovative teaching/learning with geotechnologies in secondary education. In A Tatnall \& T. Jones (éd.) Proceedings of the IFIP Conference. : Springer.

\subsubsection{Pour les rapports internes et les thèses}

Tout doit être en romain.

Mercat, C., Hasek, R., Leistenschneider M., Pech, P., Recio, T., Soury-Lavergne S., Trgalová J. (2009). Report on best practises for the use of DGS content, deliverable No D6.2, 2009, november, The Intergeo Consortium, $66 \mathrm{p}$.

Sanchez, E. (2007). Investigation scientifique et modélisation pour l'enseignement des sciences de la Terre. Contribution à l'étude de la place des technologies numériques dans la conduite d'une classe de terrain au lycée, thèse de doctorat, Université de Lyon, 400 p.

\subsubsection{Pour les références sur le Web}

Les références aux documents électroniques s'inspirent des schémas présentés ci-dessus selon qu'il s'agit d'une monographie ou d'un article de périodique. En lieu et place de «l'adresse bibliographique» (Lieu : édition, année), on indique la mention «Disponible sur Internet», l'adresse électronique précise (et complète) pour $\mathrm{y}$ accéder et, entre parenthèses, la date de votre dernière consultation du document.

Robillard, J. Ontologies : antinomies, contradictions et autres difficultés épistémologiques du concept. Sticef, 2004, 11. Disponible sur Internet: <http://sticef.univ- 
lemans.fr/num/vol2004/robillard-05/sticef_2004_robillard_05.htm> (consulté le 13 avril 2005).

Quand il s'agit d'un document imprimé accessible par voie électronique, un document PDF par exemple, on indique les références usuelles, en ajoutant son importance matérielle (nombre de pages) ainsi que les mentions évoquées ci-dessus.

Larivain, C. \& Cormier, J.-Y. Portrait des enseignants de collèges et lycées : interrogation de 1000 enseignants du second degré en mai-juin 2004. Paris : ministère de l'Éducation nationale, de l'Enseignement supérieur et de la Recherche : direction de l'Évaluation et de la Prospective, 2004, $182 \mathrm{p}$. Disponible sur Internet : $<\mathrm{ftp}$ //trf.education.gouv.fr/pub/edutel/dpd/dossiers/dossier163/dossier163.pdf> (consulté le 13 avril 2005). 